Jun 2001

KEK-TH-771

\title{
Closed String Tachyons in Non-supersymmetric Heterotic Theories
}

\author{
Takao Suyama \\ Theory Group, KEK \\ Tsukuba, Ibaraki 305-0801, Japan
}

\begin{abstract}
We calculate partition functions for supersymmetric heterotic theories on Melvin background with Wilson line. These functions coincide with partition functions for some of non-supersymmetric heterotic theories in appropriate limits. This suggests that nonsupersymmetric heterotic theories are equivalent to supersymmetric theories on supersymmetrybreaking backgrounds, just as in the case of recently conjectured IIA-0A duality.
\end{abstract}

\footnotetext{
${ }^{1}$ e-mail address : tsuyama@post.kek.jp
} 


\section{Introduction}

String theory has infinitely many perturbative vacua. There are many non-supersymmetric vacua which in general contain tachyons in their spectrum, as well as supersymmetric ones. The existence of tachyons indicates an instability of the perturbative vacuum, and it has been believed that the theory becomes stable after condensing the tachyon. In recent researches [1], the fate of an open string tachyon has been well-studied. The tachyon has a nontrivial potential and the stable vacuum of the theory corresponds to the global minimum of the potential. The stabilization corresponds to the decay of an unstable D-brane system on which the open string is attached. The occurrence of this phenomenon has been verified quantitatively.

The case of closed string tachyon is rather difficult to analyze. Recently, an interesting conjecture is made in [2] [3] on the fate of closed string tachyon in Type 0A theory (see also [5]). It is argued that Type 0A theory is equivalent to Type IIA theory on Melvin background [6] with a particular value of parameters. Then the instability due to the closed string tachyon in Type 0A theory is identified with the one due to pair creation of D6- $\overline{\mathrm{D} 6}$, and it is argued that the theory becomes Type IIA theory after closed string tachyon condensation [2] [3]. This picture seems to be correct, so it is natural to expect that such understanding can be applicable to other theories containing closed string tachyons.

In this paper, we consider various non-supersymmetric heterotic theories [7]. All such theories but one have a closed string tachyon, so these are good places to examine the range of applicability of the above picture.

Type IIA-Type 0A duality is based on the following facts. Type 0A theory can be obtained by twisting Type IIA by $(-1)^{F_{s}}$, where $F_{s}$ is the spacetime fermion number. Moreover there is a Type IIA orbifold which interpolates between Type IIA and Type 0A [8] [9]. The corresponding investigations in heterotic theory have already done [10]. And in [2], it is shown that the partition function of the orbifold coincides with that of Type IIA theory on the Melvin background. Therefore it is natural to expect that there are some backgrounds of heterotic theory which are dual to non-supersymmetric heterotic theories.

This paper is organized as follows. The Melvin background and its relation to Type $0 \mathrm{~A}$ theory is reviewed in section 2. The main piece of evidence of IIA-0A duality is briefly shown in section 3 . In section 4 , we calculate partition functions for supersymmetric heterotic theories on the Melvin background with several patterns of Wilson lines, and show that the partition functions coincide with those for some of non-supersymmetric heterotic theories. Section 5 is devoted to discussions. 


\section{Kaluza-Klein Melvin Background}

Consider the following spacetime in M-theory [2],

$$
d s_{11}^{2}=\eta_{\mu \nu} d x^{\mu} d x^{\nu}+d r^{2}+r^{2}(d \theta+q d y)^{2}+d y^{2}
$$

with $\mu, \nu=0,1, \cdots, 7$. $(r, \theta)$ are polar coordinates of 8-9 plane and $y$-direction is compactified on $S^{1}$. That is,

$$
\begin{gathered}
y \sim y+2 \pi m R, \\
\theta \sim \theta+2 \pi n, \\
m, n \in \mathbf{Z},
\end{gathered}
$$

where $R$ is the radius of the $S^{1}$ and $q$ is a real parameter. This metric is locally flat, so it is expected that this is an exact background of M-theory.

Although the eleven-dimensional metric is simple, the corresponding Type IIA background is nontrivial. The ten-dimensional metric and other fields in Type IIA theory can be read off from the metric (2.1) by the well-known relation,

$$
d s_{11}^{2}=e^{-\frac{2}{3} \phi} d s_{10}^{2}+e^{\frac{4}{3} \phi}\left(d y+A_{\mu} d x^{\mu}\right)^{2} .
$$

The followings are the corresponding Type IIA background known as the Kaluza-Klein Melvin background [2].

$$
\begin{aligned}
d s_{10}^{2} & =\sqrt{1+q^{2} r^{2}}\left(\eta_{\mu \nu} d x^{\mu} d x^{\nu}+d r^{2}\right)+\frac{r^{2}}{\sqrt{1+q^{2} r^{2}}} d \theta^{2} \\
e^{\frac{4}{3} \phi} & =1+q^{2} r^{2} \\
A_{\theta} & =\frac{q r^{2}}{1+q^{2} r^{2}}
\end{aligned}
$$

Since the dilaton diverges as $r \rightarrow \infty$, this is not a perturbative background. The only nonzero component of the R-R field strength is

$$
F_{89}=\frac{2 q}{\left(1+q^{2} r^{2}\right)} .
$$

This indicates that the R-R flux is localized around $r=0$. The total flux is finite:

$$
\frac{1}{2 \pi} \int_{\mathbf{R}^{2}} F=\frac{1}{q}
$$

This configuration is called a fluxbrane or a F7-brane [3]. Further investigations on the fluxbranes can be found in [4]

Surprisingly, there is a periodicity in $q$ [2]. This periodicity is rather apparent in the eleven-dimensional solution, although it seems difficult to find such a property from the 
ten-dimensional one. It is convenient to introduce new coordinate $\tilde{\theta}=\theta+q y$. Then the identifications of the coordinates (2.2) become as follows.

$$
\begin{aligned}
& y \sim y+2 \pi m R \\
& \tilde{\theta} \sim \tilde{\theta}+2 \pi n+2 \pi m q R
\end{aligned}
$$

One can see that when $q=\frac{k}{R}$ ( $k$ is an integer), $\tilde{\theta}$ is just the ordinary angular variable. Therefore the corresponding spacetime is $\mathbf{R}^{10} \times S^{1}$ which is the same as the one with $q=0$.

If there exist spacetime fermions, the situation is a bit complicated. Suppose that there is a fermion whose boundary condition is periodic along the $S^{1}$. When $q=\frac{1}{R}$, the parallel transport of the fermion along the $S^{1}$ is accompanied by the $2 \pi$ rotation in the 8-9 plane. So the boundary condition is antiperiodic at this special value of $q$. When

$q=\frac{2}{R}$, the boundary condition is again periodic because of the $4 \pi$ rotation in the 8-9 plane, and thus this background is indeed equivalent to the one with $q=0$. Therefore the period of $q$, in the presence of fermions, is $\frac{2}{R}$.

The background with $q=\frac{1}{R}$, when compactified to ten dimensions, corresponds to Type 0A theory [9]. Type 0A theory is a theory of closed strings which contain a tachyonic mode. The existence of such a closed string tachyon would indicate an instability of the vacuum, just as in the case of open string tachyon [1]. The correspondence discussed above means that Type 0A theory can be interpreted as Type IIA theory on the particular background. Based on this point of view, it is argued that the instability due to the closed string tachyon in Type 0A theory corresponds, in Type IIA theory, to the one due to pair creation of D6-branes [2] [3] (instability of the Melvin background is investigated in [11]). After the closed string tachyon is stabilized in some manner, Type 0A theory would become Type IIA theory.

\section{Duality between Type IIA and Type 0A}

The main piece of evidence of the duality between Type IIA and Type 0A is the coincidence of their partition functions on appropriate backgrounds [2]. As mentioned before, perturbative calculations are not valid in the background (2.4). In addition, it is very difficult to analyze string theory on R-R background. Fortunately, the background (2.4) can be related to a NS-NS background by further compactifying the metric (2.1) on $S^{1}$ along, say, 7-direction and reducing to ten dimensions along the $S^{1}$. The resulting Type IIA background is as follows.

$$
d s_{N S}^{2}=\eta_{\alpha \beta} d x^{\alpha} d x^{\beta}+d r^{2}+r^{2}(d \theta+q d y)^{2}+d y^{2}
$$

The indices $\alpha, \beta$ run from 0 to 6 . The dilaton is constant and there is no R-R background. 
It is shown in [12] that Type IIA theory on the background (3.1) can be reduced to a free theory, so the partition function can be calculated exactly. Note that since the background (3.1) is locally flat, the worldsheet theory is conformal for any value of $q$ [13].

The partition function for general $q$ is 12 [2]

$$
\begin{aligned}
Z_{q} \propto & \int \frac{d^{2} \tau}{\tau_{2}} \tau^{-4}|\eta(\tau)|^{-18} \sum_{m, n \in \mathbf{Z}} \exp \left(-\frac{\pi R^{2}}{\alpha^{\prime} \tau_{2}}|n+m \tau|^{2}\right) \\
& \left|\vartheta\left[\begin{array}{c}
1 / 2+m q R \\
1 / 2+n q R
\end{array}\right](0, \tau)\right|^{-2}\left|\vartheta\left[\begin{array}{c}
1 / 2+m q R / 2 \\
1 / 2+n q R / 2
\end{array}\right](0, \tau)\right|^{8} .
\end{aligned}
$$

We have used the theta function with characteristics

$$
\vartheta\left[\begin{array}{l}
a \\
b
\end{array}\right](\nu, \tau)=\sum_{n \in \mathbf{Z}} \exp \left[\pi i(n+a)^{2} \tau+2 \pi i(n+a)(\nu+b)\right] .
$$

The details of this calculation will be explained in the next section. From the above expression, one can see the periodicity in $q$ [12],

$$
Z_{q+2 / R}=Z_{q}
$$

discussed in the previous section.

Since Type IIA theory contains spacetime fermions, the shift of $q$ by $\frac{1}{R}$ is expected to make a change. In fact, the partition function becomes

$$
\begin{aligned}
Z_{1 / R} \propto & \int \frac{d^{2} \tau}{\tau_{2}} \tau_{2}^{-5}|\eta(\tau)|^{-24} \\
& \left.\left.\left(Z_{(1,0)}|\vartheta| \begin{array}{l}
1 \\
0
\end{array}\right](0, \tau)\right|^{8}+Z_{(0,1)}\left|\vartheta\left[\begin{array}{l}
0 \\
1
\end{array}\right](0, \tau)\right|^{8}+Z_{(1,1)}\left|\vartheta\left[\begin{array}{l}
0 \\
0
\end{array}\right](0, \tau)\right|^{8}\right),
\end{aligned}
$$

where

$$
Z_{(\epsilon, \delta)}=\sum_{k, l \in \mathbf{Z}} \exp \left[-\frac{\pi R^{2}}{\alpha^{\prime} \tau_{2}}|(2 k+\epsilon)+(2 l+\delta) \tau|^{2}\right] .
$$

In the $R \rightarrow 0$ limit,

$$
Z_{1 / R} \rightarrow \int \frac{d^{2} \tau}{\tau_{2}} \tau_{2}^{-5}|\eta(\tau)|^{-16}\left(\left|Z_{0}^{1}(\tau)\right|^{8}+\left|Z_{1}^{0}(\tau)\right|^{8}+\left|Z_{0}^{0}(\tau)\right|^{8}\right)
$$

where $Z_{\delta}^{\epsilon}(\tau)$ is the partition function for a complex fermion defined in [14]. The RHS of (3.7) is the partition function for Type 0A theory compactified on $S^{1}$ with vanishing radius. In the eleven-dimensional background (2.1), the $R \rightarrow 0$ limit just corresponds to Type IIA limit. Therefore (3.7) can be understood as evidence of the duality between Type IIA on the Melvin background and Type 0A. It can also be interpreted that (3.7) shows a duality between ten-dimensional theories in the T-dual picture. 


\section{Non-supersymmetric heterotic theory}

So far we have reviewed the duality between Type IIA theory on the Melvin background and Type 0A theory. This duality leads one to the conjecture on the fate of the closed string tachyon in Type 0A theory [2] [3]. Another approach to closed string tachyon has been made recently [15]. The stabilization of closed string tachyon is a phenomenon which is difficult to study, although that of open string tachyon has been well-studied [1]. Therefore it will be very helpful for understanding the fate of closed string tachyons if such a dual picture exists in general.

It is well-known that there are several non-supersymmetric heterotic theories in ten dimensions [7] and all the theories but one have tachyons in their spectrum. Thus it is natural to expect that these tachyonic theories can also be interpreted as supersymmetric heterotic theory on particular backgrounds which break all of supersymmetries. In this section we will show evidence which suggests the existence of such dual descriptions.

\subsection{Background of heterotic theory}

We consider the NS-NS Melvin background (3.1) and a Wilson line along the $y$-direction. It is convenient to rewrite the metric as follows.

$$
d s_{N S}^{2}=\eta_{\mu \nu} d x^{\mu} d x^{\nu}+|d x+i q x d y|^{2}+d y^{2}
$$

We have introduced a complex coordinate $x$,

$$
x=r e^{i \theta}=x^{7}+i x^{8} .
$$

Then the bosonic part of the worldsheet action of heterotic theory on this background is 12

$$
S_{b}=\frac{1}{4 \pi \alpha^{\prime}} \int d^{2} \sigma\left[\eta_{\mu \nu} \partial_{a} X^{\mu} \partial_{a} X^{\nu}+\left|\partial_{a} X+i q \partial_{a} y X\right|^{2}+\partial_{a} y \partial_{a} y\right]
$$

For the right-moving fermions, we employ the Green-Schwarz formalism. Their action is 12

$$
S_{\text {right }}=\frac{i}{\pi} \int d^{2} \sigma \bar{S}_{R}^{r}\left(\partial_{+}+\frac{i}{2} q \partial_{+} y\right) S_{R}^{r} .
$$

with $r=1, \cdots, 4$. Note that the coefficient of $\partial_{+} y$ is half of that in the bosonic part.

The action of the left-moving fermions is not affected by the Melvin background. Instead, by the presence of the Wilson line the action is as follows.

$$
S_{l e f t}=\frac{i}{\pi} \int d^{2} \sigma \sum_{I=1}^{16} \bar{\lambda}_{L}^{I}\left(\partial_{-}-i A_{y}^{I} \partial_{-} y\right) \lambda_{L}^{I}
$$

As mentioned in the previous section, this theory is conformal for any value of $q$ (and $\left.A_{y}^{I}\right)$. 
At first sight, the above action is too complicated to analyze, due to the nontrivial coupling between $y$ and other fields. However, as shown in [12], this can be reduced to a free theory. The reduction is accomplished by the following field redefinitions.

$$
\begin{aligned}
& X=e^{-i q y} \tilde{X} \\
& S_{R}^{r}=e^{-\frac{i}{2} q y} \tilde{S}_{R}^{r} \\
& \lambda_{L}^{I}=e^{i A_{y}^{I} y} \tilde{\lambda}_{L}^{I}
\end{aligned}
$$

The Jacobian of this redefinition is trivial. The action of $\tilde{X}, \tilde{S}_{R}^{r}$ and $\tilde{\lambda}_{L}^{I}$ becomes the free one. The existence of the nontrivial background is encoded in the boundary conditions.

$$
\begin{aligned}
& y(\sigma+2 \pi)=y(\sigma)+2 \pi m R \\
& \tilde{X}(\sigma+2 \pi)=e^{2 \pi i m q R} \tilde{X}(\sigma) \\
& \tilde{S}_{R}^{r}(\sigma+2 \pi)=e^{\pi i m q R} \tilde{S}_{R}^{r}(\sigma) \\
& \tilde{\lambda}_{L}^{I}(\sigma+2 \pi)=e^{-2 \pi i m R A_{y}^{I}} \tilde{\lambda}_{L}^{I}(\sigma)
\end{aligned}
$$

The torus partition function of this theory can be easily calculated, for example, by the operator formalism. For a complex boson with boundary conditions

$$
\begin{aligned}
& \phi\left(\sigma^{1}+2 \pi, \sigma^{2}\right)=e^{2 \pi i \theta_{1}} \phi\left(\sigma^{1}, \sigma^{2}\right), \\
& \phi\left(\sigma^{1}+2 \pi \tau_{1}, \sigma^{2}+2 \pi \tau_{2}\right)=e^{-2 \pi i \theta_{2}} \phi\left(\sigma^{1}, \sigma^{2}\right),
\end{aligned}
$$

the partition function is $Z_{1,2 \theta_{2}}^{1,2 \theta_{1}}(\tau)^{-1}$, where

$$
Z_{1,2 \theta_{2}}^{1,2 \theta_{1}}(\tau) \propto \vartheta\left[\begin{array}{c}
1 / 2+\theta_{1} \\
1 / 2+\theta_{2}
\end{array}\right](0, \tau) \eta(\tau)^{-1}
$$

and $\tau$ is the modulus of the torus. Similarly, for a complex fermion with boundary conditions

$$
\begin{aligned}
& \psi\left(\sigma^{1}+2 \pi, \sigma^{2}\right)=-e^{2 \pi i \theta_{1}} \psi\left(\sigma^{1}, \sigma^{2}\right), \\
& \psi\left(\sigma^{1}+2 \pi \tau_{1}, \sigma^{2}+2 \pi \tau_{2}\right)=-e^{-2 \pi i \theta_{2}} \psi\left(\sigma^{1}, \sigma^{2}\right),
\end{aligned}
$$

the partition function is $Z_{0,2 \theta_{2}}^{0,2 \theta_{1}}(\tau)$.

In the case of heterotic theories, the phases of the partition functions of the fermions are important. They are determined in [16] by an argument of anomaly. The precise expression, including the phases, of the partition functions is as follows.

$$
\begin{aligned}
& Z_{0,2 \theta_{2}}^{0,2 \theta_{1}}(\tau)=e^{-\pi i \theta_{1} \theta_{2}} \vartheta\left[\begin{array}{l}
\theta_{1} \\
\theta_{2}
\end{array}\right](0, \tau) \eta(\tau)^{-1} \\
& Z_{1,2 \theta_{2}}^{0,2 \theta_{1}}(\tau)=e^{-\pi i \theta_{1}\left(\theta_{2}+1\right)} \vartheta\left[\begin{array}{c}
\theta_{1} \\
1 / 2+\theta_{2}
\end{array}\right](0, \tau) \eta(\tau)^{-1} \\
& Z_{0,2 \theta_{2}}^{1,2 \theta_{1}}(\tau)=e^{-\pi i \theta_{1} \theta_{2}} \vartheta\left[\begin{array}{c}
1 / 2+\theta_{1} \\
\theta_{2}
\end{array}\right](0, \tau) \eta(\tau)^{-1} \\
& Z_{1,2 \theta_{2}}^{1,2 \theta_{1}}(\tau)=-i e^{-\pi i \theta_{1}\left(\theta_{2}+1\right)} \vartheta\left[\begin{array}{c}
1 / 2+\theta_{1} \\
1 / 2+\theta_{2}
\end{array}\right](0, \tau) \eta(\tau)^{-1}
\end{aligned}
$$


Here P (A) indicates (anti-)periodic boundary condition, so, for example, (AP) means antiperiodic along $\sigma^{1}$ direction and periodic along $\sigma^{2}$ direction. The modular property of these functions is summarized in Appendix A.

Now one can obtain the partition function for supersymmetric heterotic theories on the NS-NS Melvin background with the Wilson line,

$$
\begin{aligned}
& Z_{q}\left(A_{y}^{I}\right) \\
= & \int \frac{d^{2} \tau}{\tau_{2}} \tau_{2}^{-4}|\eta(\tau)|^{-12} \sum_{m, n \in \mathbf{Z}} \exp \left[-\frac{\pi R^{2}}{\alpha^{\prime} \tau_{2}}|n+m \tau|^{2}\right]\left|Z_{1,2 n q R}^{1,2 m q R}(\tau)\right|^{-2} Z_{l e f t}(\tau) Z_{1, n q R}^{1, m q R}(\tau)^{*}
\end{aligned}
$$

where

$$
Z_{\text {left }}(\tau)=\prod_{I=1}^{16} Z_{0,2 n R A_{y}^{I}}^{0,2 m R A_{y}^{I}}(\tau)+\prod_{I=1}^{16} Z_{1,2 n R A_{y}^{I}}^{0,2 m R A_{I}^{I}}(\tau)+\prod_{I=1}^{16} Z_{0,2 n R A_{y}^{I}}^{1,2 m R A_{y}^{I}}(\tau)+\prod_{I=1}^{16} Z_{1,2 n R A_{y}^{I}}^{1,2 m R A_{y}^{I}}(\tau)
$$

for the $S O(32)$ theory, and

$$
\begin{aligned}
Z_{\text {left }}(\tau)=\prod_{k=0,1} & \left(\prod_{I=1+8 k}^{8+8 k} Z_{0,2 n R A_{y}^{I}}^{0,2 m R A_{y}^{I}}(\tau)+\prod_{I=1+8 k}^{8+8 k} Z_{1,2 n R A_{y}^{I}}^{0,2 m R A_{y}^{I}}(\tau)\right. \\
& \left.+\prod_{I=1+8 k}^{8+8 k} Z_{0,2 n R A_{y}^{I}}^{1,2 m R A_{y}^{I}}(\tau)+\prod_{I=1+8 k}^{8+8 k} Z_{1,2 n R A_{y}^{I}}^{1,2 m R A^{I}}(\tau)\right)^{2}
\end{aligned}
$$

for the $E_{8} \times E_{8}$ theory.

\subsection{Coincidence between partition functions}

We evaluate the above partition function (4.19) at $q=\frac{1}{R}$ in the $R \rightarrow 0$ limit with the following Wilson lines;

(i) $A_{y}^{I}=\left(1,0^{15}\right) / R$,

(ii) $A_{y}^{I}=\left(1,0^{7}, 1,0^{7}\right) / R$ in $E_{8} \times E_{8}$ theory and

$$
A_{y}^{I}=\left(\left(\frac{1}{2}\right)^{8}, 0^{8}\right) / R \text { in } S O(32) \text { theory, }
$$

(iii) no Wilson line.

(i) Non-SUSY theory containing tachyons

First we consider $S O(32)$ heterotic theory. Then the partition function (4.19) is

$$
Z_{1 / R}\left(1,0^{15}\right)=\int \frac{d^{2} \tau}{\tau_{2}} \tau_{2}^{-5}|\eta(\tau)|^{-16} \sum_{m, n \in \mathbf{Z}} \exp \left[-\frac{\pi R^{2}}{\alpha^{\prime} \tau_{2}}|n+m \tau|^{2}\right] Z_{1, n}^{1, m}(\tau)^{* 4}
$$




$$
\begin{gathered}
\left(Z_{0,2 n}^{0,2 m}(\tau) Z_{0}^{0}(\tau)^{15}+Z_{1,2 n}^{0,2 m}(\tau) Z_{1}^{0}(\tau)^{15}+Z_{0,2 n}^{1,2 m}(\tau) Z_{0}^{1}(\tau)^{15}\right) \\
=\int \frac{d^{2} \tau}{\tau_{2}} \tau_{2}^{-5}|\eta(\tau)|^{-16}\left\{Z_{(1,0)}\left(Z_{0}^{0}(\tau)^{16}+Z_{1}^{0}(\tau)^{16}-Z_{0}^{1}(\tau)^{16}\right) Z_{0}^{1}(\tau)^{*^{4}}\right. \\
+Z_{(0,1)}\left(Z_{0}^{0}(\tau)^{16}-Z_{1}^{0}(\tau)^{16}+Z_{0}^{1}(\tau)^{16}\right) Z_{1}^{0}(\tau)^{*^{4}} \\
\left.+Z_{(1,1)}\left(Z_{0}^{0}(\tau)^{16}-Z_{1}^{0}(\tau)^{16}-Z_{0}^{1}(\tau)^{16}\right) Z_{0}^{0}(\tau)^{* 4}\right\}
\end{gathered}
$$

Note that the contribution from $X$ diverges when $q=\frac{1}{R}$ since the translational invariance in 8-9 plane is restored. So we have replaced $\left|Z_{0}^{0}(\tau)\right|^{-2}$ with the ordinary eta function.

This partition function is the same as that for an orbifold of $S O(32)$ theory. To see this, rewrite $Z_{(\epsilon, \delta)}$ as follows.

$$
\begin{aligned}
Z_{(\epsilon, \delta)} & =\frac{\sqrt{\alpha^{\prime} \tau_{2}}}{2 R} \sum_{k, l \in \mathbf{Z}} \exp \left[-\pi \tau_{2}\left(\frac{\alpha^{\prime}}{4 R^{2}}(2 k)^{2}+\frac{4 R^{2}}{\alpha^{\prime}}\left(l+\frac{\delta}{2}\right)^{2}\right)+2 \pi i \tau_{1}(2 k)\left(l+\frac{\delta}{2}\right)\right] \\
+(-1)^{\epsilon} & \frac{\sqrt{\alpha^{\prime} \tau_{2}}}{2 R} \sum_{k, l \in \mathbf{Z}} \exp \left[-\pi \tau_{2}\left(\frac{\alpha^{\prime}}{4 R^{2}}(2 k+1)^{2}+\frac{4 R^{2}}{\alpha^{\prime}}\left(l+\frac{\delta}{2}\right)^{2}\right)+2 \pi i \tau_{1}(2 k+1)\left(l+\frac{\delta}{2}\right)\right] \\
& \equiv \mathcal{Z}_{(0, \delta)}+(-1)^{\epsilon} \mathcal{Z}_{(1, \delta)}
\end{aligned}
$$

These are familiar summations for the momentum and winding modes, except that there are winding modes with half-integral winding numbers. Then the partition function (4.22) takes the following form.

$$
\begin{aligned}
& Z_{1 / R}\left(1,0^{15}\right) \\
& =\int \frac{d^{2} \tau}{\tau_{2}} \tau_{2}^{-5}|\eta(\tau)|^{-16}\left[\mathcal{Z}_{(0,0)}\left(Z_{0}^{0}(\tau)^{16}+Z_{1}^{0}(\tau)^{16}-Z_{0}^{1}(\tau)^{16}\right) Z_{0}^{1}(\tau)^{*^{4}}\right. \\
& -\mathcal{Z}_{(1,0)}\left(Z_{0}^{0}(\tau)^{16}+Z_{1}^{0}(\tau)^{16}-Z_{0}^{1}(\tau)^{16}\right) Z_{0}^{1}(\tau)^{*^{4}} \\
& +\mathcal{Z}_{(0,1)}\left\{\left(Z_{0}^{0}(\tau)^{16}-Z_{1}^{0}(\tau)^{16}\right)\left(Z_{0}^{0}(\tau)^{*^{4}}+Z_{0}^{1}(\tau)^{*^{4}}\right)\right. \\
& \left.+Z_{0}^{1}(\tau)^{16}\left(Z_{1}^{0}(\tau)^{*^{4}}-Z_{0}^{0}(\tau)^{*^{4}}\right)\right\} \\
& +\mathcal{Z}_{(1,1)}\left\{\left(Z_{0}^{0}(\tau)^{16}-Z_{1}^{0}(\tau)^{16}\right)\left(Z_{1}^{0}(\tau)^{*^{4}}-Z_{0}^{0}(\tau)^{*^{4}}\right)\right. \\
& \left.\left.+Z_{0}^{1}(\tau)^{16}\left(Z_{1}^{0}(\tau)^{*^{4}}+Z_{0}^{0}(\tau)^{*^{4}}\right)\right\}\right]
\end{aligned}
$$

This corresponds to an orbifold studied in $\llbracket 10$. It can be shown that such orbifold in the $R \rightarrow 0$ limit becomes non-supersymmetric $\overrightarrow{S O}(32)$ theory. In fact, by taking the limit,

$Z_{1 / R}\left(1,0^{15}\right) \rightarrow \int \frac{d^{2} \tau}{\tau_{2}} \tau_{2}^{-5}|\eta(\tau)|^{-16}\left(Z_{0}^{0}(\tau)^{16} Z_{0}^{0}(\tau)^{*^{4}}-Z_{1}^{0}(\tau)^{16} Z_{1}^{0}(\tau)^{*^{4}}-Z_{0}^{1}(\tau)^{16} Z_{0}^{1}(\tau)^{*^{4}}\right)$

which is the partition function of non-supersymmetric $S O(32)$ theory [14]. 
Similar result can be obtained in $E_{8} \times E_{8}$ theory. In this case, the partition function is

$$
\begin{array}{r}
Z_{1 / R}\left(1,0^{15}\right)=\int \frac{d^{2} \tau}{\tau_{2}} \tau_{2}^{-5}|\eta(\tau)|^{-16} Z_{E_{8}}(\tau)\left\{Z_{(1,0)}\left(Z_{0}^{0}(\tau)^{8}+Z_{1}^{0}(\tau)^{8}-Z_{0}^{1}(\tau)^{8}\right) Z_{0}^{1}(\tau)^{*^{4}}\right. \\
+Z_{(0,1)}\left(Z_{0}^{0}(\tau)^{8}-Z_{1}^{0}(\tau)^{8}+Z_{0}^{1}(\tau)^{8}\right) Z_{1}^{0}(\tau)^{*^{4}} \\
\left.+Z_{(1,1)}\left(Z_{0}^{0}(\tau)^{8}-Z_{1}^{0}(\tau)^{8}-Z_{0}^{1}(\tau)^{8}\right) Z_{0}^{0}(\tau)^{*^{4}}\right\} \\
\rightarrow \int \frac{d^{2} \tau}{\tau_{2}} \tau_{2}^{-5}|\eta(\tau)|^{-16} Z_{E_{8}}(\tau)\left(Z_{0}^{0}(\tau)^{8} Z_{0}^{0}(\tau)^{*^{4}}-Z_{1}^{0}(\tau)^{8} Z_{1}^{0}(\tau)^{*^{4}}-Z_{0}^{1}(\tau)^{8} Z_{0}^{1}(\tau)^{*^{4}}\right)
\end{array}
$$

where

$$
Z_{E_{8}}(\tau)=Z_{0}^{0}(\tau)^{8}+Z_{1}^{0}(\tau)^{8}+Z_{0}^{1}(\tau)^{8}
$$

Therefore the resulting theory is non-supersymmetric $E_{8} \times S O(16)$ theory.

(ii) Non-SUSY theory without tachyon

Non-supersymmetric $S O(16) \times S O(16)$ theory can also be interpreted as supersymmetric heterotic theory on the Melvin background. Consider first $E_{8} \times E_{8}$ theory. The partition function (4.19) is then

$$
\begin{aligned}
Z_{1 / R}\left(1,0^{7}, 1,0^{7}\right) & \\
=\int \frac{d^{2} \tau}{\tau_{2}} \tau_{2}^{-5}|\eta(\tau)|^{-16} & \left\{Z_{(1,0)}\left(Z_{0}^{0}(\tau)^{8}+Z_{1}^{0}(\tau)^{8}-Z_{0}^{1}(\tau)^{8}\right)^{2} Z_{0}^{1}(\tau)^{* 4}\right. \\
+ & Z_{(0,1)}\left(Z_{0}^{0}(\tau)^{8}-Z_{1}^{0}(\tau)^{8}+Z_{0}^{1}(\tau)^{8}\right)^{2} Z_{1}^{0}(\tau)^{* 4} \\
& \left.-Z_{(1,1)}\left(Z_{0}^{0}(\tau)^{8}-Z_{1}^{0}(\tau)^{8}-Z_{0}^{1}(\tau)^{8}\right)^{2} Z_{0}^{0}(\tau)^{*^{4}}\right\} \\
\rightarrow \int \frac{d^{2} \tau}{\tau_{2}} \tau_{2}^{-5}|\eta(\tau)|^{-16} & \left\{\left(Z_{0}^{0}(\tau)^{8}+Z_{1}^{0}(\tau)^{8}-Z_{0}^{1}(\tau)^{8}\right)^{2} Z_{0}^{1}(\tau)^{*^{4}}\right. \\
+ & \left(Z_{0}^{0}(\tau)^{8}-Z_{1}^{0}(\tau)^{8}+Z_{0}^{1}(\tau)^{8}\right)^{2} Z_{1}^{0}(\tau)^{*^{4}} \\
- & \left.\left(Z_{0}^{0}(\tau)^{8}-Z_{1}^{0}(\tau)^{8}-Z_{0}^{1}(\tau)^{8}\right)^{2} Z_{0}^{0}(\tau)^{* 4}\right\}
\end{aligned}
$$

in the $R \rightarrow 0$ limit. The contribution from fermions to the partition function (i.e. the terms inside the curly bracket) can be rewritten as follows.

$$
\begin{aligned}
& 2\left\{Z_{0}^{0}(\tau)^{8}-Z_{1}^{0}(\tau)^{8}\right\} Z_{0}^{1}(\tau)^{8}\left\{Z_{0}^{0}(\tau)^{*^{4}}+Z_{1}^{0}(\tau)^{*^{4}}\right\} \\
+ & {\left[\left\{Z_{0}^{0}(\tau)^{8}+Z_{1}^{0}(\tau)^{8}\right\}^{2}+Z_{0}^{1}(\tau)^{16}\right]\left\{Z_{0}^{0}(\tau)^{*^{4}}-Z_{1}^{0}(\tau)^{*^{4}}\right\} } \\
- & {\left[\left\{Z_{0}^{0}(\tau)^{8}-Z_{1}^{0}(\tau)^{8}\right\}^{2}+Z_{0}^{1}(\tau)^{16}\right] Z_{0}^{1}(\tau)^{*^{4}} } \\
- & 2\left\{Z_{0}^{0}(\tau)^{8}+Z_{1}^{0}(\tau)^{8}\right\} Z_{0}^{1}(\tau)^{8} Z_{0}^{1}(\tau)^{*^{4}}
\end{aligned}
$$


Therefore the partition function (4.28) exactly coincides with that for non-supersymmetric $S O(16) \times S O(16)$ theory (see, e.g. [10]).

The same partition function can be obtained from supersymmetric $S O(32)$ theory.

$$
\begin{aligned}
& Z_{1 / R}\left(\left(\frac{1}{2}\right)^{8}, 0^{8}\right) \\
\rightarrow & \int \frac{d^{2} \tau}{\tau_{2}} \tau_{2}^{-5}|\eta(\tau)|^{-16}\left\{2 Z_{0}^{0}(\tau)^{8} Z_{1}^{0}(\tau)^{8} Z_{0}^{1}(\tau)^{* 4}\right. \\
& \left.\quad+2 Z_{0}^{0}(\tau)^{8} Z_{0}^{1}(\tau)^{8} Z_{1}^{0}(\tau)^{*^{4}}-2 Z_{1}^{0}(\tau)^{8} Z_{0}^{1}(\tau)^{8} Z_{0}^{0}(\tau)^{* 4}\right\}
\end{aligned}
$$

One can easily see that this is the same quantity as (4.28).

It seems interesting that there exist such dual descriptions for $S O(16) \times S O(16)$ theory which has no tachyon in the spectrum. In the IIA-0A case, it is argued that the instability of the Melvin background in IIA picture is identified with the one due to the presence of tachyon in $0 \mathrm{~A}$ picture [2] [3]. So it is interesting to identify the instability due to the Melvin background in terms of $S O(16) \times S O(16)$ theory. It is known that although $S O(16) \times S O(16)$ theory does not have tachyon at all, the perturbative vacuum of this theory is unstable due to a quantum effect [7]. Thus the dual descriptions might enable one to understand such instability.

(iii) Supersymmetric theory

The final example is supersymmetric theory on the Melvin background without Wilson line. Then the contribution to the partition function from the left-moving fermions is the ordinary one; $Z_{S O(32)}(\tau)$ or $Z_{E_{8}}(\tau)^{2}$, where

$$
Z_{S O(32)}(\tau)=Z_{0}^{0}(\tau)^{16}+Z_{1}^{0}(\tau)^{16}+Z_{0}^{1}(\tau)^{16}
$$

The partition function (4.19) in this case is

$$
Z_{1 / R}\left(0^{16}\right) \rightarrow \int \frac{d^{2} \tau}{\tau_{2}} \tau_{2}^{-5}|\eta(\tau)|^{-16} Z_{\text {left }}(\tau)\left\{Z_{0}^{1}(\tau)^{*^{4}}+Z_{1}^{0}(\tau)^{*^{4}}-Z_{0}^{0}(\tau)^{*^{4}}\right\}
$$

in the $R \rightarrow 0$ limit. Therefore this suggests that a supersymmetric theory on the critical Melvin background is equivalent to the original supersymmetric theory. This is a new feature of this kind of duality, which does not occur in Type IIA case. The spacetime fermions emerge from the twisted sector, if the theory is interpreted as the orbifold $S O(32)$ or $E_{8} \times E_{8}$ on $S^{1} /(-1)^{F_{s}} \cdot \sigma^{\frac{1}{2}}$. 


\section{Discussions}

We have investigated the duality between supersymmetric heterotic theories on special backgrounds and nonsupersymmetric heterotic theories. This is based on the fact that the partition functions of two theories coincide. It is necessary to find further evidence which supports this duality conjecture. Fortunately, now we know some nonperturbative aspects of string theory. The duality web may be useful to study the properties of such backgrounds. For example, Type IIA-heterotic duality or Type I-heterotic duality will help us understand a mechanism which stabilizes the closed string tachyons. It will also be important to understand the instability in heterotic theories from the eleven-dimensional point of view, as has been done in [2].

In heterotic theories, the Melvin background corresponds to various perturbative vacua which are determined by Wilson line. However the stability property of the vacua is not common among them. The typical examples are; (i) unstable vacuum with tachyons, (ii) unstable vacuum without tachyon, (iii) stable vacuum. The instability of the vacua will be due to that of the Melvin background, so it is interesting to clarify how such a rich pattern can be realized by the presence of Wilson line.

One of the important notion to understand the open string tachyon condensation is that this phenomenon can be described by a worldsheet theory. In particular, the interpretation of tachyon condensation by a RG flow induced by a boundary perturbation has provided an exact treatment [17] of this phenomenon. It will be very helpful that closed string tachyon condensation can also be understood in terms of a RG flow. Surprisingly, string theory on the Melvin background defines a family of CFT which interpolates between a theory with tachyon and another theory without tachyon. So there exists an exactly marginal deformation which connects the above two theories. Such a deformation will be related to the change in the flux, in terms of supersymmetric theory. Then the interpretation of the deformation in terms of non-supersymmetric theory will be important to understand the closed string tachyon.

What we have discussed so far is a non-supersymmetric version of the target space duality. This resembles T-duality and Mirror symmetry in the sense that two different string theories on different backgrounds define the same theory. It is very interesting to know whether this is a special case of a more general duality, although the analysis will be difficult due to the absence of supersymmetry. There may be another examples of this kind of duality.

In particular, it is interesting if there exists the following situation; there is a string theory which is dual to a background of another string theory, but the background is not connected with the trivial one in the space of solutions of the latter theory. Then it can be considered that the two theories are related by an off-shell deformation. In this situation, if exists, a nonperturbative effect will make a drastic change in the theory. It may force us to reconsider the connectivity of the moduli space of string theory. 


\section{Acknowledgements}

I would like to thank S. Iso and H. Itoyama for valuable discussions.

\section{A Modular property of partition functions}

In this appendix, we summarize the modular properties of various functions. The basic function is the theta function with characteristics.

$$
\begin{aligned}
\vartheta\left[\begin{array}{l}
a \\
b
\end{array}\right](\nu, \tau) & =\sum_{n \in \mathbf{Z}} \exp \left[\pi i(n+a)^{2} \tau+2 \pi i(n+a)(\nu+b)\right] \\
& =\exp \left[\pi i a^{2} \tau+2 \pi i a(\nu+b)\right] \vartheta_{3}(\nu+a \tau+b \mid \tau)
\end{aligned}
$$

The modular property of this function with $\nu=0$ is as follows.

$$
\begin{aligned}
& \vartheta\left[\begin{array}{l}
a \\
b
\end{array}\right](0, \tau+1)=e^{-\pi i a(a+1)} \vartheta\left[\begin{array}{c}
a \\
a+b+1 / 2
\end{array}\right](0, \tau) \\
& \vartheta\left[\begin{array}{l}
a \\
b
\end{array}\right]\left(0,-\frac{1}{\tau}\right)=(-i \tau)^{\frac{1}{2}} e^{2 \pi i a b} \vartheta\left[\begin{array}{c}
b \\
-a
\end{array}\right](0, \tau)
\end{aligned}
$$

Note that this function is periodic in $a$ and $b$, up to phase.

$$
\begin{aligned}
& \vartheta\left[\begin{array}{c}
a+1 \\
b
\end{array}\right](0, \tau)=\vartheta\left[\begin{array}{l}
a \\
b
\end{array}\right](0, \tau) \\
& \vartheta\left[\begin{array}{c}
a \\
b+1
\end{array}\right](0, \tau)=e^{2 \pi i a} \vartheta\left[\begin{array}{l}
a \\
b
\end{array}\right](0, \tau)
\end{aligned}
$$

Our main interest is the partition functions (4.18). Due to the phase factors, the modular property of the functions is so simple.

$$
\begin{gathered}
Z_{0,2 \theta_{2}}^{0,2 \theta_{1}}(\tau+1)=e^{-\frac{\pi}{12} i} Z_{1,2\left(\theta_{1}+\theta_{2}\right)}^{0,2 \theta_{1}}(\tau), \quad Z_{1,2 \theta_{2}}^{0,2 \theta_{1}}(\tau+1)=e^{-\frac{\pi}{12} i} Z_{0,2\left(\theta_{1}+\theta_{2}\right)}^{0,2 \theta_{1}}(\tau) \\
Z_{0,2 \theta_{2}}^{1,2 \theta_{1}}(\tau+1)=e^{\frac{\pi}{6} i} Z_{0,2\left(\theta_{1}+\theta_{2}\right)}^{1,2 \theta_{1}}(\tau), \quad Z_{1,2 \theta_{2}}^{1,2 \theta_{1}}(\tau+1)=e^{\frac{\pi}{6} i} Z_{1,2\left(\theta_{1}+\theta_{2}\right)}^{1,2 \theta_{1}}(\tau) \\
Z_{0,2 \theta_{2}}^{0,2 \theta_{1}}\left(-\frac{1}{\tau}\right)=Z_{0,-2 \theta_{1}}^{0,2 \theta_{2}}(\tau), \quad Z_{1,2 \theta_{2}}^{0,2 \theta_{1}}\left(-\frac{1}{\tau}\right)=Z_{0,-2 \theta_{1}}^{1,2 \theta_{2}}(\tau) \\
Z_{0,2 \theta_{2}}^{1,2 \theta_{1}}\left(-\frac{1}{\tau}\right)=Z_{1,-2 \theta_{1}}^{0,2 \theta_{2}}(\tau), \quad Z_{1,2 \theta_{2}}^{1,2 \theta_{1}}\left(-\frac{1}{\tau}\right)=e^{-\frac{\pi}{2} i} Z_{1,-2 \theta_{1}}^{1,2 \theta_{2}}(\tau)
\end{gathered}
$$

One can easily see that the partition function (4.19) is indeed modular invariant. 


\section{References}

[1] For a review, see e.g. A.Sen, Non-BPS States and Branes in String Theory, hepth/9904207.

[2] M.S.Costa, M.Gutperle, The Kaluza-Klein Melvin Solution in M-theory, JHEP 0103 (2001) 027, hep-th/0012072.

[3] M.Gutperle, A.Strominger, Fluxbranes in String Theory, hep-th/0104136.

[4] P.M.Saffin, Gravitating Fluxbranes, gr-qc/0104014;

M.S.Costa, C.A.R.Herdeiro, L.Cornalba, Flux-branes and the Dielectric Effect in String Theory, hep-th/0105023.

[5] J.G.Russo, A.A.Tseytlin, Magnetic Backgrounds and Tachyonic Instabilities in Closed Superstring Theory and M-theory, hep-th/0104238.

[6] M.A.Melvin, Phys. Lett. 8 (1964) 65.

[7] L.J.Dixon, J.A.Harvey, String Theories in Ten Dimensions Without Spacetime Supersymmetry, Nucl. Phys. B274 (1986) 93;

L.Alvarez-Gaume, P.Ginsparg, G.Moore, C.Vafa, An $O(16) \times O(16)$ Heterotic String, Phys. Lett. B171 (1986) 155;

N.Seiberg, E.Witten, Spin Structures in String Theory, Nucl. Phys. B276 (1986) 272 ;

H.Kawai, D.C.Lewellen, S.-H.H.Tye, Classification of Closed-fermionic-string Models, Phys. Rev. D34 (1986) 3794.

[8] R.Rohm, Spontaneous Supersymmetry Breaking in Supersymmetric String Theories, Nucl. Phys. B237 (1984) 553;

J.J.Atick, E.Witten, The Hagedorn Transition and the Number of Degrees of Freedom of String Theory, Nucl. Phys. B310 (1988) 291;

M.B.Green, Wilson-Polyakov Loops for Critical Strings and Superstrings at Finite Temperature, Nucl. Phys. B381 (1992) 201.

[9] O.Bergman, M.R.Gaberdiel, Dualities of Type 0 Strings, JHEP 9907 (1999) 022, hep-th/9906055.

[10] H.Itoyama, T.R.Taylor, Supersymmetry Restoration in the Compactified $O(16) \times$ O(16)' Heterotic String Theory, Phys. Lett. B186 (1987) 129;

J.D.Blum, K.R.Dienes, Duality without Supersymmetry: The Case of the SO(16) × SO(16) String, Phys. Lett. B414 (1997) 260, hep-th/9707148; Strong/Weak Coupling Duality Relations for Non-Supersymmetric String Theories, Nucl. Phys. B516 (1998) 83, hep-th/9707160. 
[11] F.Dowker, J.P.Gauntlett, D.A.Kastor, J.Traschen, Pair Creation of Dilaton Black Holes, Phys. Rev. D49 (1994) 2909, hep-th/9309075;

F.Dowker, J.P.Gauntlett, S.B.Giddings, G.T.Horowitz, On Pair Creation of Extremal Black Holes and Kaluza-Klein Monopoles, Phys. Rev. D50 (1994) 2662, hepth/9312172; The Decay of Magnetic Fields in Kaluza-Klein Theory, Phys. Rev D52 (1995) 6929, hep-th/9507143; Nucleation of P-branes and Fundamental Strings, Phys. Rev. D53 (1996) 7115, hep-th/9512154.

[12] J.G.Russo, A.A.Tsytlin, Closed Superstrings in Magnetic Flux Tube Background, Nucl. Phys. Proc. Suppl. 49 (1996) 338, hep-th/9510041; Magnetic Flux Models in Superstring Theory, Nucl. Phys. B461 (1996) 131, hep-th/9508068.

[13] G.T.Horowitz, A.A.Tseytlin, A New Class of Exact Solutions in String Theory, Phys. Rev. D51 (1995) 2896, hep-th/9409021;

J.G.Russo, A.A.Tseytlin, Constant Magnetic Field in Closed String Theory: An Exactly Solvable Model, Nucl. Phys. B448 (1995) 293, hep-th/9411099.

[14] J.Polchinski, String Theory vol.I 8 II, Cambridge University Press.

[15] A.Adams, E.Silverstein, Closed String Tachyons, AdS/CFT, and Large N QCD, hepth/0103220.

[16] K.S.Narain, M.H.Sarmadi, E.Witten, A Note on Toroidal Compactification of Heterotic String Theory, Nucl. Phys. B279 (1987) 369.

[17] E.Witten, On Background Independent Open String Theory, Phys. Rev. D46 (1992) 5467, hep-th/9208027; Some Computations in Background Independent Open-String Field Theory, Phys. Rev. D47 (1993) 3405, hep-th/9210065;

A.Gerasimov, S.Shatashvili, On Exact Tachyon Potential in Open String Field Theory, JHEP 0010 (2000) 034, hep-th/0009103;

D.Kutasov, M.Marino, G.Moore, Some Exact Results on Tachyon Condensation in String Field Theory, JHEP 0010 (2000) 045, hep-th/0009148. 